\title{
ENERGY DISSIPATION IN MEDIUM AND HIGH CYCLE FATIGUE OF METALLIC AND COMPOSITE MATERIALS
}

\author{
G. Meneghetti*, M. Quaresimin ${ }^{\circ}$ \\ *Department of Mechanical Engineering, University of Padova \\ via Venezia, 1, 35131 Padova, Italy \\ ${ }^{\circ}$ Department of Management and Engineering, University of Padova \\ Stradella S. Nicola, 3, 36100 Vicenza, Italy
}

Experimental estimation of the fatigue limit of metallic materials on the basis of temperature measurements is well documented in the literature [1]. Typically, infrared cameras are adopted in order to monitor surface temperature of specimens and components subjected to fatigue tests, thus leading to the so called "thermographic method". In summary, the thermographic methodology is sketched in fig. 1. When a force-controlled, constant amplitude fatigue test is running, temperature measured at the specimen surface is seen to increase at the beginning of the test and then, after a certain number of cycle $\mathrm{N}$, stabilises so that it is possible to identify a stable temperature increase $\Delta \mathrm{T}_{\text {stat }}$. The higher the stress amplitude $\sigma_{\mathrm{a}}$ the higher the stable temperature increase, as depicted in fig. 1a. Since fatigue failure is caused by microplastic strains which initiate a small fatigue crack, then temperature increments have been qualitatively interpreted as the manifestation of energy dissipation due to plastic hysteresis energy. A quantitative evaluation is reported in [2]. By plotting the stable temperature increments as a function of the applied stress amplitude, one can extrapolate the $\Delta \mathrm{T}_{\text {stat }}$ versus $\sigma_{\mathrm{a}}$ curve, as shown in fig. $1 \mathrm{~b}$. By so doing, one can estimate the fatigue limit $\sigma_{0 \text {,th }}$ of the material, according to the thermographic method presented in the literature (for example [1,3] and references quoted therein).
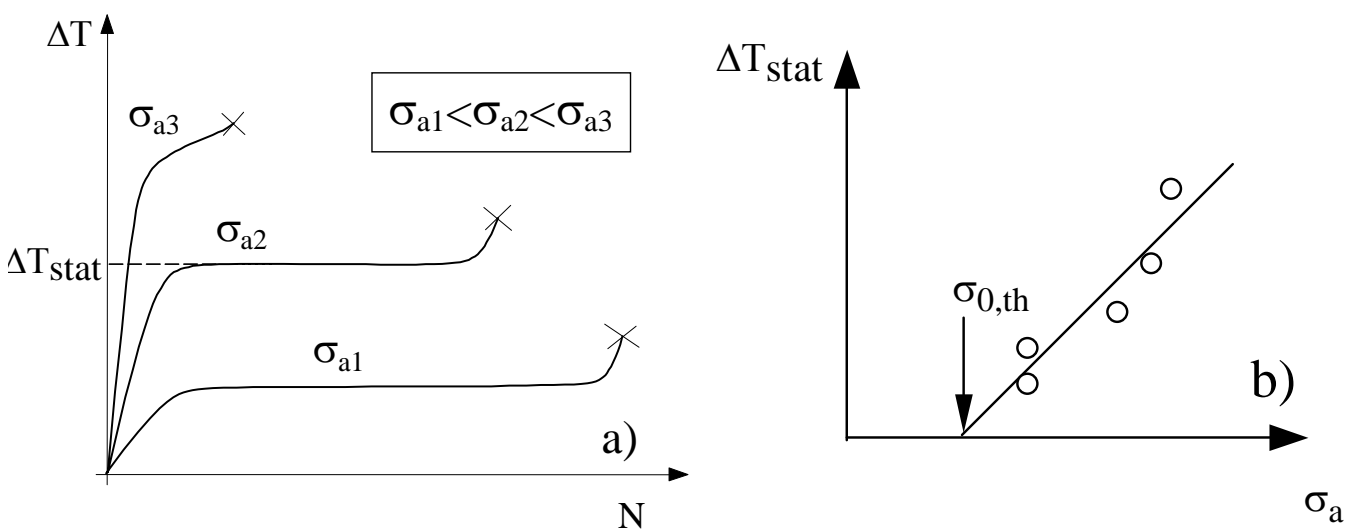

Figure 1. Observed temperature evolution during constant amplitude fatigue tests on metallic and composite materials (a), estimation of the material fatigue limit according to [1] (b).

Anyway the specific energy Q dissipated as heat in a unit volume of material per cycle seems to be a more promising parameter for fatigue characterisation. In fact, thermal increments for a given material undergoing a fatigue test depends on applied stress amplitude, test frequency, load ratio, specimen geometry and thermal boundary conditions which determine the rate of heat extraction from the material in the form of conduction, convection and radiation. Conversely, the specific energy dissipated as heat is a material parameter in constant amplitude fatigue tests, which depends only on the applied stress amplitude and load 
ratio. In particular it does not depend on the specimen geometry so that it seems reasonable to extend the laboratory test results obtained from specimens to real components having an arbitrary geometry. Recently it has been shown that the specific energy Q can be estimated by means of a simple experimental technique [4]: if the fatigue test is suddenly stopped after that temperature has reached its stable value, then the specific energy Q can be estimated from the cooling rate observed after test stopping:

$$
\left.\frac{\rho \cdot \mathrm{c}}{\mathrm{f}} \cdot \frac{\partial \mathrm{T}}{\partial \mathrm{t}}\right|_{\mathrm{t}=\left(\mathrm{t}^{*}\right)+}=-\mathrm{Q}
$$

where $\mathrm{f}$ is the test frequency, $\rho$ is the material density and $\mathrm{c}$ is the material specific heat. Aim of the present work is to show that the energy parameter Q, derived from temperature measurements by means of an infrared camera, is able to rationalise the fatigue strength of specimens having different geometries. Fig. 2 shows the results of fatigue tests obtained from

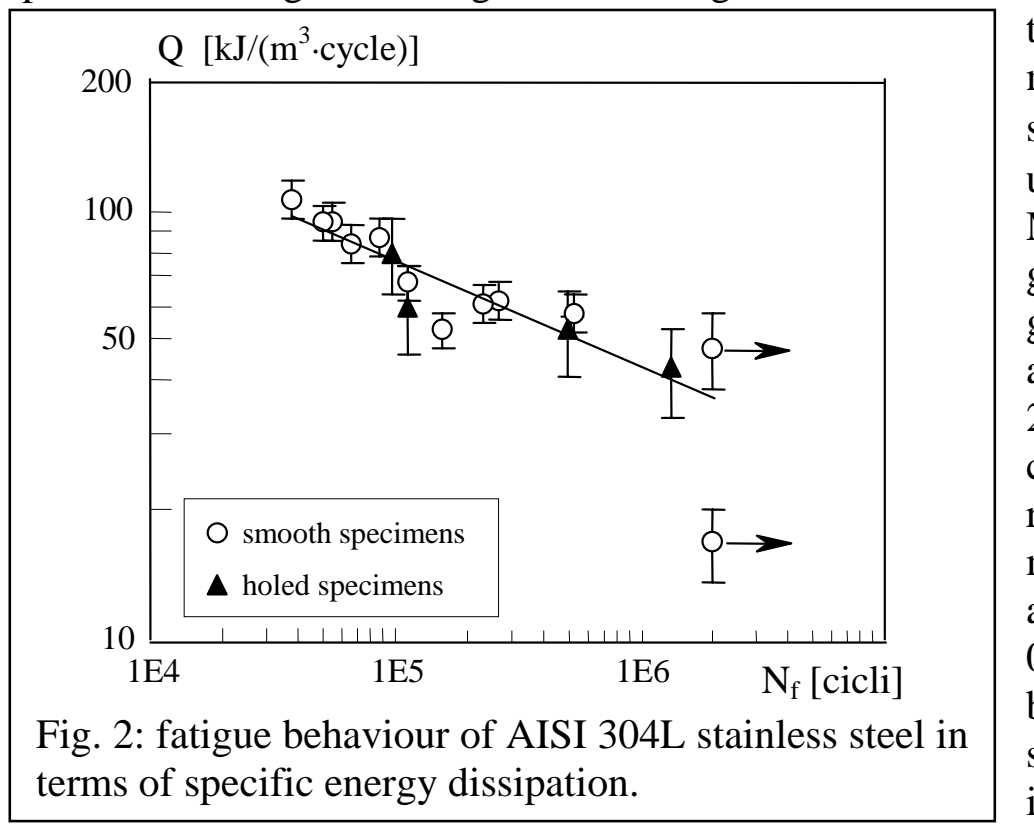
two different series of specimens made of AISI 304L stainless steel (yield stress $\sigma_{\mathrm{y}}=300 \mathrm{MPa}$, ultimate tensile stress $\sigma_{\mathrm{u}}=650$ MPa) having different geometries, i.e. a smooth geometry and a plate containing a central hole with a diameter of 20mm. Fatigue tests were conduced with a nominal stress ratio $\mathrm{R}$ (ratio between the minimum and the maximum applied nominal stress) equal to 0.1 . Fig. 2 shows that results can be summarised in terms of specific energy dissipation independently of the specimens geometry. Such an experimental technique will be extended also to a short fibre reinforced plastic. Material will be a PA66-GF35 short glass fiber polyamid composite.

Two series having different geometries will be fatigue tested at a nominal stress ratio equal to 0.1 and during fatigue tests surface temperatures will be measured by means of an infrared camera. Then by means of eq. (1) the experimental data will be processed in order to plot the fatigue lives in terms of specific energy $Q$ and to verify if $Q$ is a unifying energy parameter even for this class of materials .

\section{References}

[1] G. La Rosa, A. Risitano, Thermographic methodology for rapid determination of the fatigue limit of materials and mechanical components, Int. J. Fatigue, 22, 65-73 (2000).

[2] J. Kaleta, R. Blotny, H. Harig, Energy stored in a specimen under fatigue limit loading conditions, JTEVA, 19(4), 326-333 (1990).

[3] M.P. Luong, Fatigue limit evaluation of metals using an infrared thermographic technique, Mechanics of Materials, 28, 155-163 (1998).

[4] B. Atzori, G. Meneghetti, Energy dissipation in low cycle fatigue of austempered ductile irons, $5^{\text {th }}$ Int. Conf. on Low Cycle Fatigue, Berlin (Germany), 2003, pp. 147-152. 